\title{
The spectral form factor is not self-averaging
}

\author{
R. E. Pranged \\ Laboratoire de Physique Quantiqued, Université Paul Sabatier, \\ Toulouse, France \\ (June 25, 1996)
}

\begin{abstract}
The spectral form factor, $k(t)$, is the Fourier transform of the two level correlation function $C(x)$, which is the averaged probability for finding two energy levels spaced $x$ mean level spacings apart. The average is over a piece of the spectrum of width $W$ in the neighborhood of energy $\epsilon_{0}$. An additional ensemble average is traditionally carried out, as in random matrix theory. Recently a theoretical calculation of $k(t)$ for a particular system, with an energy average only, found interesting nonuniversal semiclassical effects at times $t \sim 1$ in units of $\tau_{\hbar}=2 \pi \hbar /$ (mean level spacing). This is of great interest if $k(t)$ is self-averaging, i.e, if the properties of a typical member of the ensemble are the same as the ensemble average properties. We here argue that this is not always the case, and that for many important systems an ensemble average is essential to see detailed properties of $k(t)$. In other systems, notably the Riemann zeta function, it is likely possible to see the properties by an analysis of the spectrum.
\end{abstract}

Recent work [1] connects the energy level statistics of a particular system, say a Bunimovich stadium billiard, with the predictions of random matrix theory [RMT] [2]. This work has generated great interest and some controversy. In particular it was suggested that the form factor $k(t)$ of the spectrum of a given system can be calculated theoretically to good approximation directly in terms of certain classical parameters [i.e. the periodic orbits] of the system. For scaling systems like billiards, not only does the form factor approach the universal RMT result in an appropriate high energy limit, but predictions [1], [3] are made as to how this limit is approached. These predictions differ in detail from one another, but their gross features are the same.

It would obviously be of interest to take the spectrum of some system, obtained either numerically or experimentally, and use it to calculate $k(t)$, so that comparison of the approximate theories with the numerically 'exact' structure factor can be made. The main result of this paper is that in many cases such a calculation is unexpectedly difficult, and may even be impossible in principle. We refer to this difficulty as the fact that the form factor is not a self-averaging quantity.

Hard chaotic billiards without time reversal symmetry, 'GUE' [2], the case worked out in detail up to now, unfortunately fall into the probably impossible category. Billiards with nonisolated orbits are likely to be difficult although not impossible to study numerically. However, the Riemann zeta function, on the hypothesis that its ze- roes are eigenenergies of some linear Hermitean operator, can probably be studied effectively, especially in view of the large database of zeroes obtained by Odlysko [4].

Of all the two level correlation functions, [which are interrelated by linear transformations], the form factor is of particular interest because, according to the above ideas [which originated with Berry [5]], the dimensionless time $t$, the argument of $k(t)$, is essentially the relevant classical orbit period in units of the Heisenberg time, $\tau_{\hbar}=2 \pi \hbar / \Delta$, where $\Delta$ is the mean level spacing. In particular, for small times $t$, it has long been established [and exploited [6]] that the form factor is strongly peaked at the periods of the short periodic orbits, and the weights of these peaks are determined by the stability properties of the orbits. Other correlators smear over the time and are not so directly interpreted classically.

The new feature of the above theories is that there are definite nonuniversal effects in a particular range of $t$, namely near $t=1$. These effects disappear in the high energy limit, as some power of energy. Exactly which power determines whether the effects can be observed in numerical calculations.

The form factor is usually loosely defined as the Fourier transform of the two level correlation function $C(x)$,

$C(x)=\Delta^{2}\left\langle\sum_{a, b} \delta\left(E+\frac{1}{2} x \Delta-E_{a}\right) \delta\left(E-\frac{1}{2} x \Delta-E_{b}\right)\right\rangle_{W}$

where the spectrum is given by the sequence of levels $E_{a}$. The average of $E$ is over an energy range $W$ about a central energy $\epsilon_{0}$. An additional average historically has been over an ensemble of systems, for example ones given by RMT or by a random scattering potential. We are investigating the consequences of disposing of this second average. The form factor is then

$$
k(t)=\int d x e^{2 \pi i x t} C(x)
$$

Two quite different techniques have been used. One [1] starts from a supersymmetric integral representation of $C(x)$, and then makes some approximations which are discussed and justified. The other [3] extends the diagonal approximation [5] in an imaginative way to all values of $t$. In both cases it is assumed that the energy average by itself validates the operation used to give the diagonal approximation, [DA], [for $t<<1$ ] which is equivalent to the idea that the actions of all orbits [unrelated 
by symmetry] may be separately averaged. In addition, statistics of classical orbits are invoked in the form of the Hannay-Ozorio de Almeida sum rule [7], and possible extensions of it [1].

A third technique [8] explicitly combines random matrix theory [2] with orbital information about a particular system. It therefore does make an ensemble average, something like an average over the random class of billiards all with the same area and also whose short orbits have the same classical periods and stabilities. The results are less complete, but do agree qualitatively with the other methods.

Ensemble averages are often used as a calculational device, even when no ensemble is actually contemplated. The resistance of a particular copper wire is conveniently calculated by averaging over an ensemble representing the 'random' position of impurities. An experimentalist can check the theory by measuring the resistance of just one wire, however. That's because resistance properties of a typical member of the ensemble are the same as the average resistance over the ensemble: resistance is in this case a self-averaging quantity. Self-averaging is similar to ergodicity. For an ergodic system, a time-averaged quantity is self-averaging.

Clearly self-averaging is a valuable trait. It is therefore disappointing to find that the spectral form factor, by one standard definition, is not self-averaging. Other definitions can be used, and some of them are self-averaging. However, the alternative definitions known to us are defective in the sense that in important cases they eliminate the predicted effects: they are not sufficiently flexible to extract the small predicted effects from the spectral data.

The energy average is essential, [even with an ensemble average], but we are interested in the case that the results depend only weakly on the averaging window $W$, and indeed, they should not depend much on exactly how the average is made. This is supposed to be the case if the following two facts hold.

- There are a large number of energy levels in the average, i.e. $w \equiv W / \Delta>>1$.

- The averaging width $W$ is classically small. This means that energy dependent objects like the mean level spacing, and other quantities characterizing the underlying classical system, do not vary much, [or can be linearized], in the energy range $\epsilon_{0} \pm \frac{1}{2} W$. For short we describe this as $W<<\epsilon_{0}$, which tacitly supposes a natural zero of the energy.

Theorists like to clean things up by taking a limit: $\epsilon_{0} / W \rightarrow \infty, W / \Delta \rightarrow \infty$. This requires a system with an infinite number of levels, and also requires knowledge of how things change as $\epsilon_{0}$ gets very large. A prominent example of such a system is that of billiards. The order of limits is not completely trivial however and another parameter is needed. Such limits should be left to the very end, and then scrutinized carefully. In fact, numeri- cal calculations can't take such limits, so one must study how they deviate from the limiting behavior.

The energy average alone certainly suffices to give interesting results for small $t, t \simeq t_{c}$, where $t_{c}$ is the scale of the periods of the shortest periodic orbits measured in units of the Planck time. For such short times, $k(t)$ gives the length spectrum [6], that is, it has peaks at $t=T_{p} / \tau_{\hbar}$, i.e. at the periods of the short periodic orbits, which for billiards are proportional to the length of the orbits.

Here is a more careful definition. Let the energy average be given by a Gaussian, i.e. $\langle f(E)\rangle_{W}=$ $\frac{2}{\sqrt{\pi} W} \int d E f(E) \exp \left(-4\left(E-\epsilon_{0}\right)^{2} / W^{2}\right)$. We argue next that the $x$ integral in Eq.(2) should be cut off, and we choose a Gaussian: $\exp \left(-\left(x \Delta / W_{x}\right)^{2}\right)$. We claim that a natural choice is $W_{x} \sim W$, and the particular choice $W_{x}=W$ is especially congenial.

Clearly $W_{x}$ should not be too large, since approximations relying on $x \Delta<<\epsilon_{0}$ must be made. If $W$ is as large as possible, then $W_{x}$ can't be much bigger. There is no advantage to taking $W$ unnecessarily small, as one would be throwing away data, in a numerical calculation, for no gain. So, we take $W_{x} \leq W$.

The choice $W_{x}<<W$ has virtue. However, the same effect can be achieved by making a time-convolution on $k(t)$, i.e. smearing each $t$ by an amount $\tau$. We find it convenient to choose this route. Therefore, we define

$$
k(t)=\int_{-\infty}^{\infty} d x e^{2 \pi i x t} e^{-\frac{x^{2}}{w^{2}}} C(x)
$$

where $C$ is based on the Gaussian energy average.

This $k(t)$ [except for $|t|<<1$ ] does not look like the ensemble average. Rather, it has very considerable 'noise' around the ensemble mean value.

To see this, we remark that $k(t)$ can be written

$$
k(t)=\left|\sum_{a} F_{a} e^{2 \pi i \frac{E_{a}}{\Delta}} t\right|^{2}
$$

where $F_{a}^{2}=\frac{2 \Delta}{\sqrt{\pi} W} \exp \left(-4\left(E_{a}-\epsilon_{0}\right)^{2} / W^{2}\right)$. The particular choice of cutoff $W_{x}=W$ has the advantage that the absolute square of a single sum appears, rather than requiring a double sum. It indicates, for example, that $k(t)$ should be close to a positive function, no matter the form of the averaging. More general functions, $F_{a}$, appropriately normalized, will do just as well. Note that for large $t$, one expects just the diagonal terms in the double sum to survive, so that in some sense $k \rightarrow 1$ in this limit. At very small $t$, it is expected that $k(t) \simeq \delta(t)$. But in this case, the sum can be replaced by an integral, and a Gaussian of width $\Delta / W$ replaces the $\delta$ function.

Eq.(14) makes it clear that for the spectrum of a chaotic system, $k(t)$, for large $t$, is approximately given by a random walk in the complex plane, of about $w$ steps. For the kind of systems envisaged, there will be significant random walk character even for $t \sim 1$. Insofar as the 
phases of the individual terms in the sum Eq.(何) are indeed independent random variables, one may show that the distribution of values of $y=k(t)$ is

$$
\rho(y)=e^{-y} .
$$

This result is independent of the form of $F_{a}$ and of $W$, as long as there are many terms in the sums of Eq.(1), i.e. as long as $w>>1$. Basically, the result is an application of the central limit theorem. The mean value [over an ensemble of such random variables] is $\langle k(t)\rangle=1$, and the variance of the distribution is $\left\langle(k(t)-1)^{2}\right\rangle=1$.

For smaller $t$, think of the $E_{a}$ 's as ordered. Then it will take some number of steps, of order $g(t)$, say, before the knowledge of the original phase is lost. There will then be $w / g(t)$ effective random walk steps. The effective step length is still of order unity, because the phase changes systematically by an amount $e^{2 \pi i t}$ at each step. This gives a formula for the distribution of $k(t), \rho(k(t))=$ $g(t) e^{-k(t) g(t)}$. A natural guess for $g(t)$ gives

$$
\rho(k(t))=\frac{1}{k_{E}(t)} e^{-k(t) / k_{E}(t)},
$$

where $k_{E}(t)$ is the ensemble average form factor.

Thus, for any averaging scale $W$, the calculated value of $k(t)$ will suffer large fluctuations, when $t$ is of order unity or greater. Increasing $W$ does not change the distribution, but it does make $k(t)$ vary more rapidly. It's clear that if $t$ is changed by an amount of order $\Delta / W$ then $k(t)$ changes appreciably. For example, one estimates the random walk average of $\left\langle\left|\frac{d}{d t} \sum_{a} F_{a} e^{2 \pi i t\left(E_{a}-\epsilon_{0}\right) / \Delta}\right|^{2}\right\rangle=$ $\frac{4 \pi^{2}}{\Delta^{2}} \sum_{a}\left(E_{a}-\epsilon_{0}\right)^{2} F_{a}^{2} \sim(\pi w)^{2}$.

From this it follows that smearing the time $t$ by an amount $\tau$ is like averaging over a number $\tau W / \Delta$ independent choices from the distribution $\rho(k(t))$. Thus the 'noise' in the smeared function $k_{\tau}(t)$ is reduced by a factor $\sqrt{1 / w \tau}$ and of course $k_{\tau}(t)$ changes appreciably only when $t$ is varied by amount of order $\tau$.

There is literature which numerically displays the noise and time averages in $k(t)$ just discussed. A recent Letter [9] studies the spectrum of hydrogen in a magnetic field. The distribution $\rho(y)$ is not mentioned, although the scaling suggested in Eq.(6) is tacitly used in taking the time average, [over $\tau \sim 0.6$ ]. The results agree qualitatively with the discussion we have presented. There is a calculation [10] of the statistics of the eigenphases of an $N \times N$ scattering matrix $S$, for a particular quantum chaotic scattering. The function corresponding to $k(t)$ is $N^{-1}\left|\operatorname{Tr} S^{t N}\right|^{2}$ where $t$ is a discrete time, $t=n / N$, and $t=1$ is the Heisenberg time. In this case there is an ensemble of such systems which was used to help to reduce the noise.

In the limit $\tau w \rightarrow \infty$ the noise disappears, and $k_{\tau}(t)$ in that limit becomes self-averaging. For some purposes this is adequate. For example Pandey [11, 'proved' that 'all' spectral correlation functions of particular members of an ensemble of random matrices (and thus presumably $k(t)$ ), are self-averaging [or 'ergodic']. Of course, $C(x)$ as defined in Eq.(11) is manifestly not self-averaging, since it consists of lots of $\delta$-functions, while the ensemble average is smooth. But Pandey argued that "observable" quantities involve an integral over $x$. Presumably the limits of such an integral are fixed and independent of $\epsilon_{0}$. This is energy smearing rather than time smearing. Then in the limit $\epsilon_{0} \rightarrow \infty$ followed by $W \rightarrow \infty$, Pandey indeed finds the energy smeared $C(x) \rightarrow$ random matrix prediction. Now one could, if desired, define $k(t)$ as the Fourier transform of this $C(x)$. But since the large $W$ limit is taken first, for fixed finite $x$, it's the same as doing a time average with $\tau W / \Delta \rightarrow \infty$.

We are more ambitious. Based on references [1], [3], [8], we predict for GUE billiards, that $\left|\langle k(t)\rangle-k_{R}(t)\right| \sim$ $1 / \sqrt{\epsilon_{0}}$, for $|t-1| \sim 1 / \sqrt{\epsilon_{0}}$. An ensemble average of $k$ is indicated and $k_{R}$ is the random matrix form factor. Here units are chosen so that $\Delta=1$, mass $=1 / 2$, and $\hbar=1$.

We therefore consider the 'renormalized' function $h(s) \equiv \sqrt{\epsilon_{0}}\left[k_{\tau}\left(1+s / \sqrt{\epsilon_{0}}\right)-k_{R}\left(1+s / \sqrt{\epsilon_{0}}\right)\right]$ which should approach a theoretically predictable limit, 'the signal', of order unity, as $\epsilon_{0} \rightarrow \infty$ provided we can choose a time smearing $\tau$ which leads to a $k_{\tau}(t)$ sufficiently close to the ensemble average prediction.

Put $w \simeq \epsilon_{0}^{\beta}$, and $\tau \simeq \epsilon_{0}^{-\alpha}$. Clearly $\beta \leq 1$, in order that $W<<\epsilon_{0}$ for large $\epsilon_{0}$. If the time smearing is not to lose the signal, then $\alpha \geq \frac{1}{2}$. The 'noise' in this case scales as $\epsilon_{0}^{(\alpha-\beta) / 2}$. Multiplying by $\sqrt{\epsilon_{0}}$, in order to calculate the noise in the function $h$, gives a noise $\epsilon_{0}^{\gamma}$ where $\gamma \geq \frac{1}{4}$. Thus, we predict that the noise grows at high energy, and that the signal will be swamped.

Perhaps more sophisticated data-analysis schemes could extract the signal from the noise, but all those we have tried were unsuccessful. One has to keep in mind that this is not true noise: for a given spectrum it is reproducible. Because, the noise grows at high energy in the renormalized $h(s)$, it hurts rather than helps to use very high energy data. In favorable cases, it might be possible to use low energy data, but that does not seem systematically possible. Thus, we characterize this situation as giving unexpected numerical difficulties and perhaps it is impossible in principle to check the theoretical predictions, absent an actual ensemble average.

Fortunately, there are systems which have much larger 'quasiclassical' corrections to the random matrix results, than do strongly chaotic billiards. Most notable is the spectrum of nontrivial zeroes of the Riemann zeta function. Although the hypothetical 'Hamiltonian' whose spectrum coincides with the zeroes is not known, or even proved to exist, the 'quasiclassical' parameters are known [4]. In the language used above, with $\epsilon_{0}$ having the mathematical meaning of height in the complex plane along the critical line, the mean level spacing shrinks according to $\Delta=2 \pi / \ln \left(\epsilon_{0} / 2 \pi\right)$, which we denote $\Delta \sim \epsilon_{0}^{-0}$. The 'orbit' periods in units of the Planck time are $t_{p, m}=(m \Delta / 2 \pi) \ln p$, where $p$ is prime and $m$ repe- 
titions of the fundamental orbit are taken. The stability weights, $a_{p}$ in the Gutzwiller formula, are $e^{-m \ln p / 2}$. We expect, based on the techniques of reference [8], that corrections of magnitude $\epsilon_{0}^{-0}$ over a width $|t-1| \sim \epsilon_{0}^{-0}$ will exist. There should be enough data available [ [4] to verify this prediction.

Similarly, we think that GUE billiards with strong, nonisolated orbits, will also have effects near $t=1 \mathrm{ob}-$ servable in principle, but they are probably too difficult in practice. The weight of a nonisolated orbit in the Gutzwiller formula goes as $\epsilon_{0}^{-1 / 4}$ rather than $\epsilon_{0}^{-1 / 2}$ as for unstable orbits. We expect corrections of magnitude $\epsilon_{0}^{-1 / 4}$ over a time window $\epsilon_{0}^{-1 / 4}$ thus allowing $\tau=\epsilon_{0}^{-1 / 4}$. This leads to noise of magnitude $\epsilon_{0}^{-1 / 8}$ in the rescaled $h(s)$. Thus, with many energy levels in the neighborhood of the $10^{8}$ level, such an effect might be seen in a direct calculation of $k(t)$ from the spectrum. In general, a signal of width and strength $\epsilon_{0}^{-\eta}$ can in principle be extracted from the data on a single system only if $\eta<\frac{1}{3}$.

The much studied GOE class of systems (with time reversal symmetry) unfortunately has very small effects at the Heisenberg time and should be even harder to observe. However, GSE systems, (with sympletic symmetry) might be favorable because the RMT result is singular at $t=1$ and thus could suffer large corrections from the nonuniversal effects. These two cases have not been worked out in any detail up to now, however.

Another question is whether it is possible to do ensemble averages of the type mentioned, e.g. for a class of billiards. This might work if one has an analog computer to do the job, namely an experimental system depending on a parameter in a nice way, which can be changed sufficiently to give a further average. Note that such a system is presented in reference [10].

We therefore conclude that by manipulations made on the spectrum of a specific hard chaos billiard system, it is not known how, and it is perhaps impossible, to confirm or discredit the theoretical predictions of $k(t)$ for $t \sim 1$. For other systems, particularly the Riemann zeta function, it is likely possible. The systems are distinguished by the order of magnitude of the nonuniversal effects and their dependence on energy.

Reference [1] above made no explicit ensemble or time averages, but did not find a wide distribution for $k(t)$, but rather something like the ensemble average of reference [8]. Evidently an approximation based on $W_{x} / W<<1$, was made which discarded the noise, so their results are correct if the signal has an energy exponent $\eta<\frac{1}{3}$. It's less clear what reference [3] predicts with respect to noise. Their result for $k(t)$ is a sum of $\delta$-functions, $\delta\left(t-t_{p}\right)$ at the orbit periods, but [we argue], these $\delta$-functions should have a width $\Delta / W$. Except for $t$ very small, the spacing between the $t_{p}$ 's will be much smaller than this, since the spacing decreases exponentially with increasing $t_{p}$. [This is the exponential proliferation of orbits in chaotic systems.] It was argued [5] that in this case, the Hannay-Ozorio de Almeida sum rule can be invoked with a smooth, non-noisy result. If that argument is correct and carries over to the extensions of the DA used, then these methods [3] do not produce the noise. If that is so, the noise would have to come from the neglected offdiagonal contributions. This might well be the case, as it is known that the off-diagonal contributions are very important [12]. The 'noise' in the Bogomolny-Keating formulation thus presents a deep question, which will probably not be answered in the near future.

Certain often used linear transforms of $k(t)$ are selfaveraging, in agreement with reference 111. These are $\Sigma^{2}(L)$ and $\Delta_{3}(L)$, the number variance and the DysonMehta spectral rigidity. The former is given by $\Sigma^{2}(L)=$ $\int d t k(t)[\sin \pi L t / \pi t]^{2}$. For $L \sim 1$ or smaller, there is an effective time average over a scale of order unity, thus eliminating the noise for large $W$. For large $L$, the contribution of the integral comes from small $t$, where the nonuniversal signal dominates $k(t)$.

Valuable discussions with O. Agam, E. Bogomolny, O. Bohigas, S. Fishman, B. Georgeot, J. Keating, D. Poilblanc, and C. Sire are gratefully acknowledged. We thank J. Bellissard for hospitality at the Université Paul Sabatier.

a Permanent address: Department of Physics, University of Maryland, College Park, MD 20742

b Unité Mixte de Recherche 5626

[1] O. Agam, B. L. Altshuler, and A. V. Andreev, Phys. Rev. Lett. 75, 4389 (1995); A. V. Andreev, O. Agam, B. D. Simons, and B. L. Altshuler, Phys. Rev. Lett. 76, 3947 (1996). Also see A. L. Andreev and B. L. Altshuler, Phys. Rev. Lett. 75, 902 (1995); O. Agam and S. Fishman, Phys. Rev. Lett. 76, 726 (1996).

[2] M. L. Mehta, Random Matrices, Academic Press (New York, 1990).

[3] E. B. Bogomolny and J.P. Keating, (preprint, 1996).

[4] A. M. Odlysko, Math. Comput. 48, 273-308,(1987); unpublished (1989).

[5] M. V. Berry, Proc. R. Soc. Lond. A400, 229 (1985).

[6] U. Eichmann, K. Richter, D. Wintgen and W. Sandner, Phys. Rev. Lett. 61, 2438 (1988).

[7] J.H. Hannay and A.M. Ozorio de Almeida, J. Math. Phys. A17, 3429 (1984).

[8] R. E. Prange, (submitted for publication)(May, 1996).

[9] B. Eckhardt and J. Main, Phys. Rev. Lett. 75, 2300 (1995).

[10] R. Blümel and U. Smilansky, Phys. Rev. Lett. 64, 241244 (1990).

[11] A. Pandey, Annals of Physics 119, 170-191 (1979).

[12] N. Argaman et. al., Phys. Rev. Lett. 71, 4326 (1993). 